\title{
Fetal hematopoietic stem cells express MFG-E8 during mouse embryogenesis
}

\author{
Jaehun Lee ${ }^{1}$, Byung-il Choi ${ }^{2}$, Seo Young Park $^{3}$, Su Yeon An ${ }^{1}$, Jiyou Han ${ }^{1}$ and Jong-Hoon Kim ${ }^{1}$
}

The milk fat globule-EGF-factor 8 protein (MFG-E8) has been identified in various tissues, where it has an important role in intercellular interactions, cellular migration, and neovascularization. Previous studies showed that MFG-E8 is expressed in different cell types under normal and pathophysiological conditions, but its expression in hematopoietic stem cells (HSCs) during hematopoiesis has not been reported. In the present study, we investigated MFG-E8 expression in multiple hematopoietic tissues at different stages of mouse embryogenesis. Using immunohistochemistry, we showed that MFG-E8 was specifically expressed in $\mathrm{CD} 4^{+}$HSCs at all hematopoietic sites, including the yolk sac, aorta-gonad-mesonephros region, placenta and fetal liver, during embryogenesis. Fluorescence-activated cell sorting and polymerase chain reaction analyses demonstrated that $\mathrm{CD} 34^{+}$cells, purified from the fetal liver, expressed additional HSC markers, c-Kit and Sca-1, and that these CD34+ ${ }^{+}$cells, but not CD34cells, highly expressed MFG-E8. We also found that MFG-E8 was not expressed in HSCs in adult mouse bone marrow, and that its expression was confined to $\mathrm{F} 4 / 80^{+}$macrophages. Together, this study demonstrates, for the first time, that MFG- 8 is expressed in fetal HSC populations, and that MFG-E8 may have a role in embryonic hematopoiesis.

Experimental \& Molecular Medicine (2015) 47, e174; doi:10.1038/emm.2015.42; published online 24 July 2015

\section{INTRODUCTION}

Milk fat globule-EGF-factor 8 protein (MFG-E8) is a secreted glycoprotein that has different names, SED1, BA46 and lactadherin, in different species, including mice and humans. $^{1-3}$ MFG-E8 possesses two N-terminal EGF-like domains, one of which contains a highly conserved arginine-glycine-aspartic acid (RGD) integrin-binding motif that participates in cell adhesion, by engaging $\alpha \mathrm{V} \beta 3 / 5$ integrin heterodimers. ${ }^{2,4,5}$ MFG-E8 also has two C-terminal discoidin-like domains (F5/8C domains) that mediate attachment to phosphatidylserine and phosphatidylethanolamine residues on apoptotic cells. ${ }^{6}$ Early studies of these functional domains emphasized the role of MFG-E8 as an opsonin in phagocytic clearance of apoptotic cells in various organs. ${ }^{6-11}$ However, recent studies suggest that MFG-E8 is involved in more diverse cellular events, including branching morphogenesis of the mammary gland, tissue fibrosis, repair of intestinal epithelium, angiogenesis, tumor growth and metastasis, although the underlying mechanism(s) of these events is still largely unknown. ${ }^{5,12-16}$

Hematopoietic stem cells (HSCs) have the ability to selfrenew and differentiate into all blood cell types, including blood progenitors found within the adult bone marrow. ${ }^{17}$ Bone marrow is a major postnatal hematopoietic organ that maintains HSCs after birth and throughout adult life. ${ }^{18,19}$ However, during embryogenesis, HSCs originate at a variety of anatomical sites prior to localization in the bone marrow. HSCs were first found in the yolk sac blood vessel at mouse embryonic day (E)9-10, and they localized to the aortagonad-mesonephros (AGM) at about E10.5..$^{20,21}$ At E11, HSCs then migrated to the placenta, likely through the umbilical artery. At this time point, HSC numbers in the placental labyrinth increased, particularly between the E11 and E12. ${ }^{22,23}$ It was thought that placental HSCs migrate into the fetal liver directly through the fetal blood circulation system. ${ }^{24}$ After E13.5, HSCs accumulated and expanded in the fetal liver, as the number of placental HSCs decreased. ${ }^{22}$ The HSCs then localized to the bone marrow postnatally, where they remained through adulthood.

Previous studies have demonstrated that MFG-E8 was expressed in different immune or inflammatory cells under normal or pathophysiological conditions in mice and humans. $^{7,25-28}$ However, its expression in HSCs during hematopoiesis has not yet been reported. In the present study,

\footnotetext{
${ }^{1}$ Laboratory of Stem Cells and Tissue Regeneration, Division of Biotechnology, College of Life Sciences and Biotechnology, Korea University, Seoul, Korea; ${ }^{2}$ Department of Pathology, National Cancer Center, Gyeonggi-do, Korea and ${ }^{3}$ Biomedical Research Institute, Seoul National University Hospital, Seoul, Korea Correspondence: Professor J-H Kim or Professor J Han, Laboratory of Stem Cells and Tissue Regeneration, Division of Biotechnology, College of Life Sciences and Biotechnology, Korea University, West Building/Room 304, Anam-dong 5-ga, Sungbuk-goo, Seoul 136-713, Korea.

E-mail: jhkim@korea.ac.kr or hanjiyou@hanmail.net
}

Received 23 December 2014; revised 25 February 2015; accepted 31 March 2015 
we investigated the expression of MFG-E8 in hematopoietic fetal tissues, including the yolk sac, AGM, placenta, and liver, at different stages of mouse embryogenesis. We demonstrated, for the first time, that MFG-8 was expressed in HSC populations in all hematopoietic origins, but its expression became attenuated and confined to phagocytic cells in the adult hematopoietic system.

\section{MATERIALS AND METHODS}

\section{Animals and tissue specimens}

Pregnant and adult ICR mice were purchased from the Central Laboratory (Animal Inc, Seoul, Korea). The pregnancy was confirmed by the presence of a vaginal plug. The pregnant ICR mice were killed by cervical dislocation at the following gestational ages: E9.5, E10.5, E11.5, E12.5, E13.5, E14.5, E15.5 and E16.5. Tissue specimens were obtained from at least four embryos at different implantation sites. Bone marrow was harvested from femurs of adult ICR mice (male, 6-weeks-old). Each ICR male mouse was housed in an individual cage and received sufficient food and water until it was 9-10-weeks old. Mice were killed by cervical dislocation, and femurs were obtained immediately after. Connective tissues and muscles were removed from the femurs and a crack was made with sterile scissors for fixation. All animal experiments were approved by the Institutional Animal Care and Use Committee of the Korea University (KUIACUC-2014-78).

\section{Bone marrow flushing and slide attachment}

To flush bone marrow, the femurs were first cleaned of connective tissue and muscle. Femurs were then washed with sterile phosphatebuffered saline (PBS). The bone was held in place with sterile tweezers, and the hip and knee joints were cut. The bone marrow was harvested from the proximal side of the right and left femurs. Bone marrow was flushed into a petri dish with a 26-gauge needle (Korea Vaccine, Ansan, Gyeonggi, Korea) attached to a 10-ml syringe filled with $0.5 \%$ bovine serum albumin (Bovogen, Melbourne, Australia) in PBS. The resulting cells were centrifuged at 3000 r.p.m. for $3 \mathrm{~min}$ at room temperature using a CytoSpin 4 Cytocentrifuge (Thermo, Waltham, MA, USA), then analyzed by immunostaining.

\section{Histological analyses}

Tissue specimens were fixed with $4 \%$ paraformaldehyde (Duksan, Ansan, Gyeonggi, Korea) at room temperature for at least $12 \mathrm{~h}$. The specimens were further processed by increasing ethanol concentrations (four times each in $95 \%$, and $100 \%$ for $1 \mathrm{~h}$, at room temperature), cleared in xylene (four times each, for $1 \mathrm{~h}$, at room temperature), and embedded in paraffin at $60^{\circ} \mathrm{C}$ or optimal cutting temperature compound at $-20^{\circ} \mathrm{C}$ (Tissue Tek, Chuo-ku, Tokyo, Japan). Paraffin-embedded sections of $4-10-\mu \mathrm{m}$ thickness and frozen sections $8-\mu \mathrm{m}$ thick were used. Femur sections were also decalcified using Calci-clear rapid solution (National Diagnostics, Atlanta, GA, USA) at room temperature. After deparaffinization and rehydration, the tissue sections were stained with hematoxylin (Merck, Readington Township, NJ, USA) and eosin (BBC Biochemical, Mt. Venom, WA, USA). All slides were analyzed using a Scanscope slide scanner (Aperio, Buffalo Grove, IL, USA) and ImageScope ver11.12.752 (Aperio).

\section{Immunohistochemistry}

For paraffin-embedded sections, sections of 4-10- $\mu \mathrm{m}$ thickness were dewaxed with xylene (two times, for $5 \mathrm{~min}$, at room temperature), hydrated in decreasing ethanol concentrations ( $100 \%$ two times, and
90 , 80 , and $70 \%$ once, for $3 \mathrm{~min}$, at room temperature), then with distilled water. Antigenic retrieval was performed in $10 \mathrm{mM}$ citrate buffer, at $\mathrm{pH}$ 8.0, in a microwave oven, for $10 \mathrm{~min}$. The samples were incubated at room temperature for $10 \mathrm{~min}$, after heating. After blocking with $10 \%$ goat or donkey serum in PBS, the tissue sections were incubated overnight at $4{ }^{\circ} \mathrm{C}$ with antibodies, diluted with $10 \%$ goat or donkey serum in PBS, against antibodies to CD34 (BD Biosciences, San Jose, CA, USA), MFG-E8 (MBL, Woburn, MA, USA), F4/80 (AbD Serotec, Raleigh, NC, USA) and c-Kit (Santa Cruz, Heidelberg, Germany). On the following day, the tissue sections were rinsed in PBS and incubated at room temperature for $1 \mathrm{~h}$ with appropriate secondary antibodies (Life Technologies, Carlsbad, CA, USA). Nuclei were labeled with 4,6-diamidino-2-phenylindole (DAPI; Sigma-Aldrich, St Louis, MO, USA) for $5 \mathrm{~min}$. After rinsing in $70 \%$ ethanol for $5 \mathrm{~min}$, tissue sections were treated with an autofluorescence eliminator reagent (Millipore, Billerica, MA, USA) for $5 \mathrm{~min}$ to avoid false-positive signals. Finally, tissue sections were washed and mounted with Immu-mount (Thermo), and examined with an Axiovert $200 \mathrm{M}$ fluorescence microscope (Zeiss, Oberkochen, Germany).

\section{$\mathrm{CD}^{+} 4^{+}$cell isolation}

Fetal liver tissues were harvested from 55 fetuses obtained from 6 different pregnant ICR mice at E12.5. Samples were dissociated into single cell suspensions and filtered through $100-\mu \mathrm{m}$ (BD Pharmingen, San Jose, CA, USA). To eliminate red blood cells, the cell suspension was incubated with red blood cell (RBC) lysis buffer (BD Pharmingen) for $10 \mathrm{~min}$ at room temperature, washed, and resuspended in fluorescence-activated cell sorting buffer (1\% bovine serum albumin in PBS). Samples were then labeled with anti-mouse CD34-FITC (Rat IgG, BD Pharmingen) or isotype antibody (negative control) for $20 \mathrm{~min}$ at $4{ }^{\circ} \mathrm{C}$. The cells were then separated into $\mathrm{CD} 34^{+}$and $\mathrm{CD}^{-} 4^{-}$cell fractions using a fluorescence-activated cell sortingAria (BD Pharmingen)

\section{RT-PCR and real-time PCR analyses}

Total RNA was isolated using Trizol (Invitrogen, Grand Island, NY, USA) and cDNA was synthesized from $1 \mu \mathrm{g}$ of total RNA using the RevertAid H Minus First Strand cDNA SynDissertation Kit (Thermo) according to the manufacturer's protocol. Subsequent PCR were performed using AccuPower PCR-Premix (Bioneer, Daeduck, Daejeon, Korea). Primer sequences used in this study were as shown in Table 1. PCR products were confirmed by ethidium bromide staining, following by $1 \%$ agarose gel electrophoresis. For real-time quantitative PCR (qPCR) analysis, real-time reverse transcriptionpolymerase chain reaction (RT-PCR) mixtures were performed in duplicate with the iQ SYBR Green Supermix (Bio-Rad, Hercules, CA, USA). The $\mathrm{C}_{\mathrm{T}} \mathrm{S}$ of target gene amplification were analyzed with the CFX-96 real-time PCR system (Bio-Rad). The comparative $\mathrm{C}_{\mathrm{T}}$ method $\left(2^{-\Delta \Delta \mathrm{Ct}}\right)$ was used to perform relative quantification of target genes normalized to a reference gene ( $\beta$-actin).

\section{RESULTS}

\section{MFG-E8 expression in hematopoietic tissues during} embryonic development

During mouse embryogenesis, CD34 is expressed by HSCs in different tissues, including the yolk sac, AGM, placenta and fetal liver, but its expression decreases and disappears after birth. ${ }^{29-36}$ To identify HSCs in hematopoietic tissues of staged mouse embryos, we used an HSC marker, CD34, to investigate 
the possible correlation between MFG-E8 and CD34 expression in different hematopoietic tissues. The yolk sac is the first site of primitive hematopoiesis in human and mouse embryos, and provides erythroid and myeloid cells during embryonic life. ${ }^{37}$ Hematoxylin and eosin staining of embryo sections showed that large cells with prominent nuclei were located in the yolk sac blood islands as aggregates in developing embryos (E10.5; Figures 1a-c). Immunohistochemical staining of consecutive sections of the yolk sac revealed that these cells expressed MFG-E8 (Figure 1d). Furthermore, strong expression of MFG-E8 was specifically detected in the CD34 ${ }^{+}$ HSCs, which showed a high nuclei/cytoplasm ratio (Figures le-g). A few cells which did not express CD34 were negative for MFG-E8 immunostaining (Figure 1g, arrowhead).

This observation prompted us to investigate whether all HSCs in fetal hematopoietic tissues, such as the placenta, aorta, and liver, expressed MFG-E8. Paraffin sections of E11.5

Table 1 Primer list used in this study

\begin{tabular}{ll}
\hline Primer name & Sequences \\
\hline Mouse Mfg-e8-F & 5'-ATCTACTGCCTCTGCCCTGA-3' \\
Mouse Mfg-e8-R & 5'-TGGTCTCGGTTTCACAGTGG-3' \\
Mouse Cd34-F & 5'-AGCGGTACAGGAGAATGCAG-3' \\
Mouse Cd34-R & 5'-ATCAGGCAGAGAGCTACCCA-3' \\
Mouse c-Kit-F & 5'-TCCTCACTCACGGGCGGATC-3' \\
Mouse c-Kit-R & 5'-TGGAGGTGGGGTGGGGAACT-3' \\
Mouse Sca-1-F & 5'-CAATGTAGCAGTTCCCAATG-3' \\
Mouse Sca-1-R & 5'-CAGGGGCTATAAAGGCAAAA-3' \\
Mouse $\beta$-actin-F & 5'-AGAGGGAAATCGTGCGTGAC-3' \\
Mouse $\beta$-actin-R & 5'-CAATAGTGATGACCTGGCCGT-3'
\end{tabular}

Abbreviations: F, forward; R, Reverse.

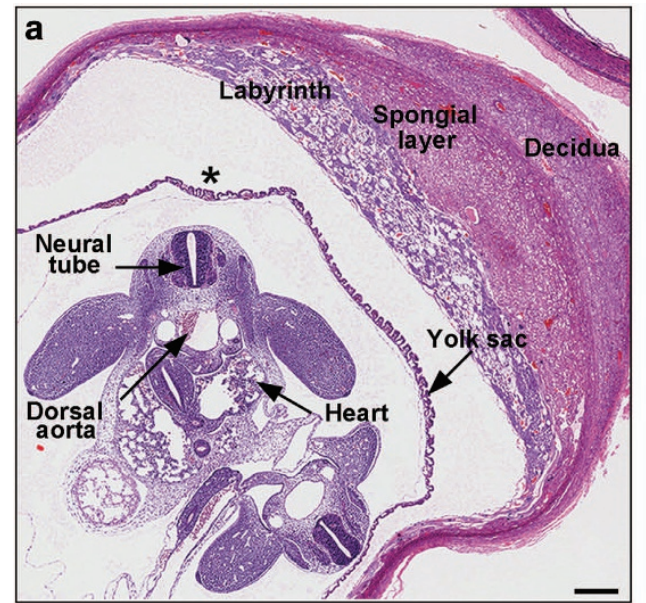

embryos were used to determine whether MFG-E8 was expressed in HSCs in different developing organs (whole hematoxylin and eosin staining images of the embryo are shown in Supplementary Figure 1). Notably, MFG-E8 expression was detected in all other hematopoietic tissues in embryos (Figures $2 \mathrm{a}-\mathrm{h}$ and $3 \mathrm{a}-\mathrm{d}$ ). We also found a few cells that did not express CD34 in hematopoietic tissues (Supplementary Figure 2). In the placenta, large blood vessels in the chorioallantoic mesenchyme of the chorionic plate contained a number of cells with large nuclei (Supplementary Figure 1 and Figure 3a, white arrowhead, inset). Immunohistochemical staining of consecutive sections of placental tissues showed that these cells were positive for CD34, and that MFG-E8 was specifically expressed in $\mathrm{CD} 34^{+}$HSCs, as observed in the yolk sac. (Figures 3b-d). There was no CD34 and/or MFG-E8 expression detected in decidua (data not shown), and only enucleated erythrocytes were found in the maternal blood vessels of the placenta (Figure 3a, black arrowhead, inset). MFG-E8 was also detected within $\mathrm{CD} 34^{+}$HSCs in the dorsal aorta and cardinal veins (E11.5; Supplementary Figure 1 and Figures 2a-d). These cells exhibited similar morphology to that of HSCs found in the yolk sac and placenta, showing a round shape with large nuclei (Supplementary Figure 1 and Figure 2a, inset), and most of these cells co-expressed CD34 and MFG-E8 (Figures 2b-d). It is believed that HSC in the yolk sac and AGM migrate to the liver at approximately day 11 , and become a main hematopoietic organ late in fetal life. ${ }^{20,24}$ At E11.5, we found many prominent colonies of eosinophilic and nucleate cells over the entire fetal liver parenchyma (Supplementary Figure 1 and Figure 2e, inset). Again, these cells expressed not only CD34, but also MFG-E8 (Figures 2f-h). Together, these results indicated that MFG-E8 was expressed in HSCs of major

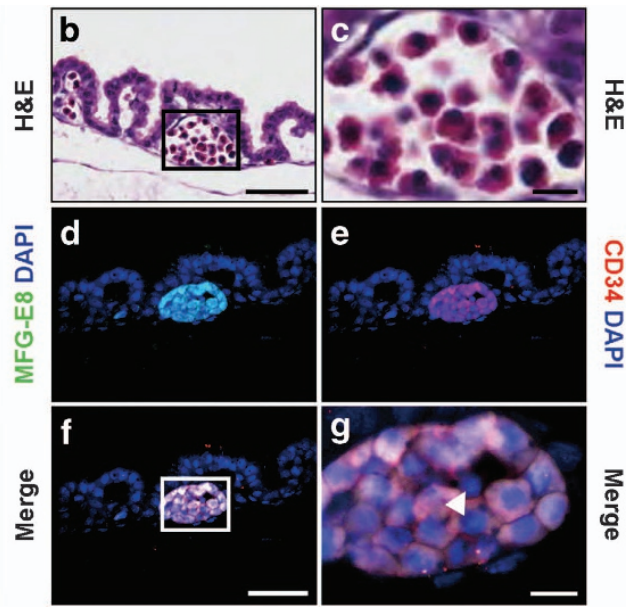

Figure 1 Milk fat globule-EGF-factor 8 (MFG-E8) and CD34 expressions in the yolk sac blood island of developing mouse fetuses at embryonic day E10.5. (a) Hematoxylin and eosin (H\&E) staining image of E10.5 mouse embryo. Decidua, spongial layer and labyrinth of the placenta are shown outside of the yolk sac surrounding the embryo. ( $\mathbf{b}$ and $\mathbf{c}$ ) Enlarged image of the area indicated with '*' in $\mathbf{a}$ are shown in b. High magnification of the boxed area in b are shown in c. Cells located in the yolk sac blood island are large and have prominent nuclei. (d-f) Immunofluorescent labeling of the blood island in the yolk sac with anti-CD34 and anti-MFG-E8 antibodies. Note that the $\mathrm{CD}_{3} 4^{+}$cell co-expressed MFG-E8. The boxed area in $\mathbf{f}$ are separately shown in $\mathbf{g}$. White arrowhead indicates CD34- cells. Scale bars in: $\mathbf{a}=250 \mu \mathrm{m} ; \mathbf{b}$ and $\mathbf{f}=50 \mu \mathrm{m}$, and $\mathbf{c}$ and $\mathbf{g}=10 \mu \mathrm{m}$. 

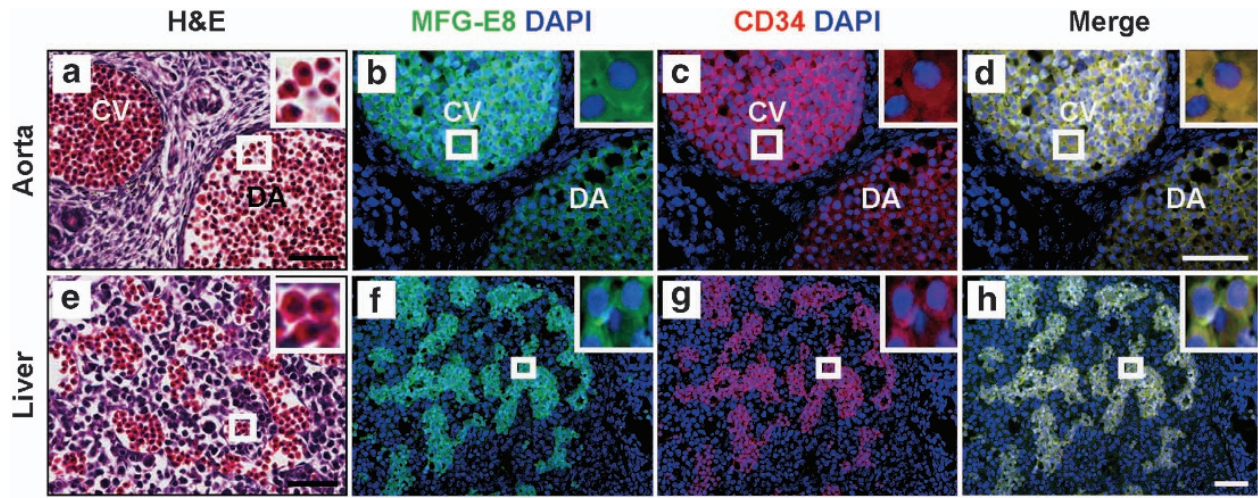

Figure 2 Milk fat globule-EGF-factor 8 (MFG-E8) and CD34 expression in the aorta, and liver of developing embryos at embryonic day E11.5 (a-d) Hematoxylin and eosin (H\&E) staining and immunofluorescent labeling of dorsal aorta and cardinal veins with anti-CD34 and anti-MFG-E8 antibodies. CV, cardinal vein; DA, dorsal aorta. (e-h) H\&E staining and immunofluorescent labeling of fetal liver sections with anti-CD34 and anti-MFG-E8 antibodies. Enlarged images of the boxed are shown in the upper right corner in each panels. Scale bar: $50 \mu \mathrm{m}$.
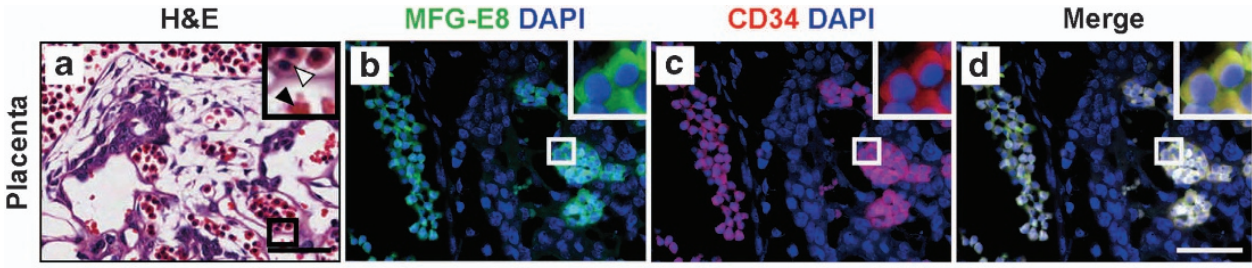

e

Embryonic day

$\begin{array}{llllll}11.5 & 12.5 & 13.5 & 14.5 & 15.5 & 16.5\end{array}$

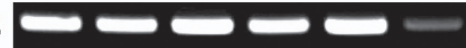

$\beta$-actin
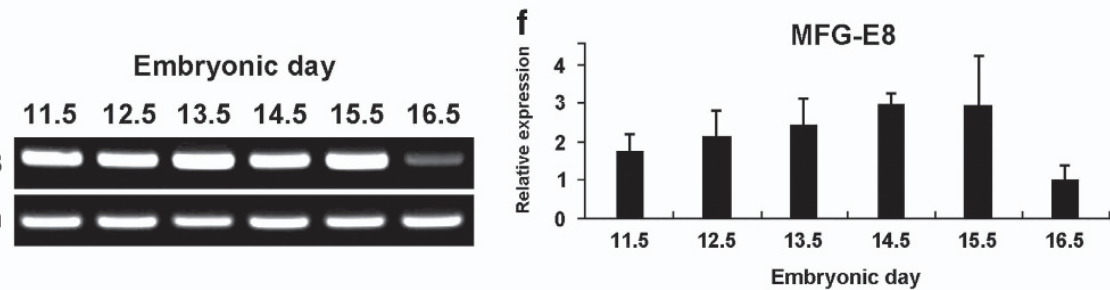

Figure 3 Expression of milk fat globule-EGF-factor 8 (MFG-E8) in placental development during pregnancy. (a-d) Hematoxylin and eosin $(\mathrm{H} \& \mathrm{E})$ staining and immunofluorescent labeling of the developing placenta with anti-CD34 and anti-MFG-E8 antibodies at embryonic day $(E) 11.5$. High magnification images of the boxed areas are shown in the upper right corner of each panels. Scale bar: $50 \mu \mathrm{m}$. (e) real-time reverse transcription-polymerase chain reaction (RT-PCR) analysis of MFG-E8 mRNA in the placenta at different days of gestation. (f) quantitative PCR (qPCR) analysis of MFG-E8 mRNA in the placenta at different days of gestation. The expression level was presented as relative expressions (fold changes) over the lowest value (the lowest level was assigned a value of 1 ) after normalization to $\beta$-actin expression.

hematopoietic tissues during the early developing phase of mouse embryos.

\section{Expression of CD34 and MFG-E8 in placental development during pregnancy}

The placenta is an important reservoir for developing and proliferating HSCs during embryogenesis. The chorionic plate acts as a hematopoietic pool before HSCs migrate to the fetal liver for further expansion. ${ }^{23,24}$ The first appearance of $\mathrm{CD} 34^{+}$ cells at the labyrinth and chorionic plate occurred at E10.5-11.5. within fetal blood vessels (Figures 3a-d). Trophoblastic cells in the labyrinth layer expressed neither CD34 nor MFG-E8. The patterns of $M f g-e 8$ expression in placental tissue was validated by RT-PCR analysis (Figure 3e). The qPCR also showed a trend of Mfg-es mRNA expression in the placenta during pregnancy, increasing gradually up to E15.5, but with marked reduction after E15.5 (Figure 3f).

\section{Expression of CD34 and MFG-E8 in the fetal liver}

The fetal liver is colonized by HSCs at days 10 and 11 of gestation, and HSC expansion initiates on E13. The fetal liver therefore serves as a main organ for HSC expansion and differentiation, until hematopoiesis in the bone marrow is established. Fetal liver HSCs have a similar surface phenotype to placental HSCs, including the hematopoietic marker CD34. ${ }^{22,23}$ We purified CD34 ${ }^{+}$cells from fetal livers at E12.5 $(n=55$ from 6 pregnant mice) to determine the selective expression of MFG-E8 in CD34 ${ }^{+}$HSCs. The CD34 antigen was expressed by $21.6 \%$ of whole-fetal liver cells after depletion of RBCs by lysis. In addition, the percentage increased up to $99 \%$ 
a
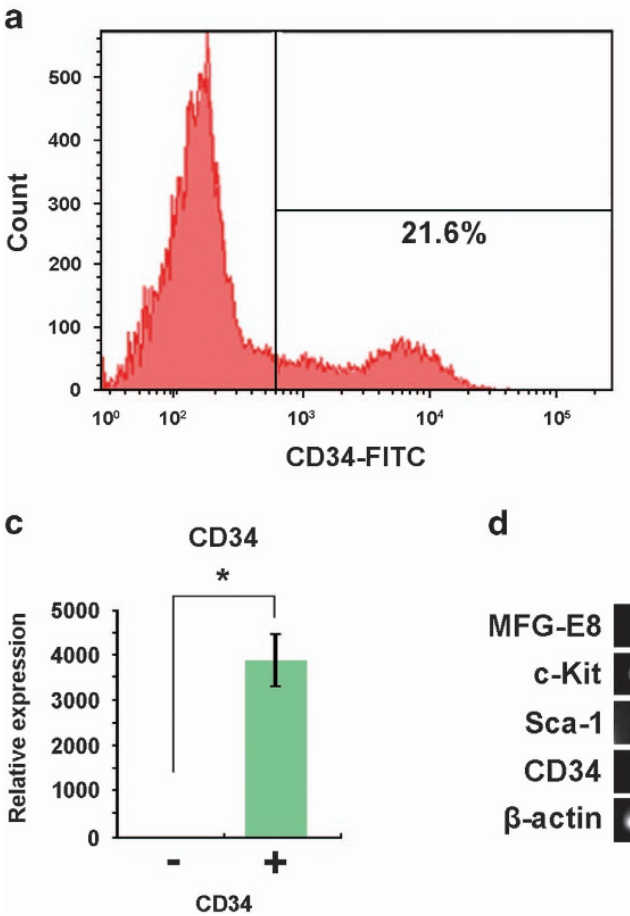

b

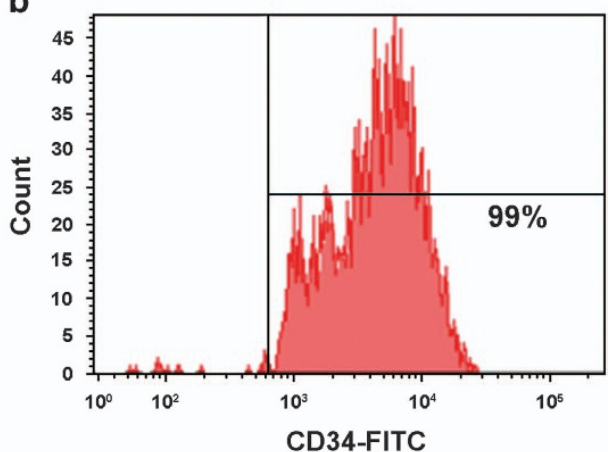

d

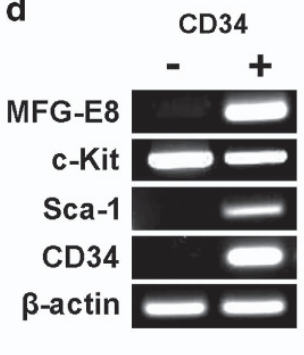

e

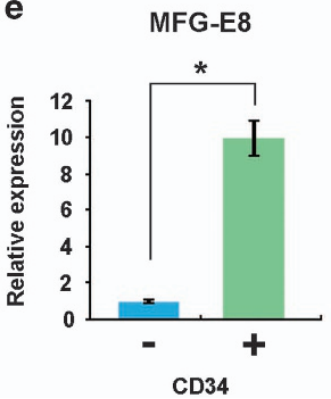

Figure 4 Expression of milk fat globule-EGF-factor 8 (MFG-E8) in CD34 ${ }^{+}$cells in the fetal liver. (a and b) Flow cytometric analysis of $\mathrm{CD}_{3}{ }^{+}$cells in the developing liver before (a) and after (b) purification with anti-CD34 antibody. (c) quantitative PCR (qPCR) analysis of sorted cells for the expression of Cd34. Cd34 expression in the CD34 ${ }^{+}$fraction was presented as relative expressions (fold changes) over CD34- fraction after normalization to $\beta$-actin expression. ${ }^{*} P<0.05$. (d and e) real-time reverse transcription-polymerase chain reaction (RT-PCR) (d) and qPCR (e) analyses of sorted CD34+ and CD34- fractions for the expression of Mfg-e8. Note that only a CD34 ${ }^{+}$ fraction expresses Mfg-e8. $P<0.05$. Mfg-e8 expression in the $\mathrm{CD} 4^{+}$fraction was presented as relative expressions (fold changes) over CD34- fraction after normalization to $\beta$-actin expression.

after purification by fluorescence-activated cell sorting, using anti-CD34 antibody (Figures $4 \mathrm{a}$ and $\mathrm{b}$ ). The purification of $\mathrm{CD}^{+} 4^{+}$cells was confirmed by qPCR, showing that $\mathrm{Cd} 34$ was expressed only in the $\mathrm{CD} 34^{+}$fraction, but not in the $\mathrm{CD} 34^{-}$ fraction (Figure 4c). Importantly, RT-PCR analysis revealed that although $c$-Kit was expressed in both $\mathrm{CD} 34^{+}$and $\mathrm{CD} 34^{-}$ fractions, Mfg-e8 expression was detected only in cells that expressed c-Kit, Sca-1 and Cd34, whereas the CD34- fraction expressed very low levels, if any, of $M f g-e 8$ (Figure 4d). The high level of $M f g-e 8$ expression in $\mathrm{CD} 4^{+}$cells was further validated by qPCR analysis (Figure 4e).

\section{CD34 and MFG-E8 expression in adult bone marrow}

To maintain the number of HSCs, hematopoiesis occurs mainly in the bone marrow throughout adult life. ${ }^{18}$ Because CD34 expression is known to be downregulated in adult HSCs, we used c-Kit, another marker of HSCs, to examine expression of MFG-E8 in mouse femur bone marrow (Supplementary Figure 3). ${ }^{38}$ Immunohistochemical analysis of bone marrow sections showed that adult $\mathrm{c}-\mathrm{Kit}^{+}$HSCs were located near the lining of sinusoids as previously reported, ${ }^{39}$ but MFG-E8 expression was not detected in ${\mathrm{c}-\mathrm{Kit}^{+}}^{+} \mathrm{HSCs}$ (Figure 5a). Because MFG-E8 expression in macrophages has been previously reported, ${ }^{13}$ we flushed bone marrow cells and assessed the expression of MFG-E8, on cytospin slides. This procedure allowed us to determine that MFG-E8 was only detected in cells expressing F4/80, a marker of mature macrophages. None of the other cells flushed out of the bone marrow expressed MFGE8 (Figure 5b).

\section{DISCUSSION}

In this study, we determined the expression pattern of MFG-E8 during embryonic hematopoiesis in mice. We found that MFG-E8 was specifically expressed in $\mathrm{CD}_{3} 4^{+}$fetal HSCs. We also showed that MFG-E8 expression was confined to $\mathrm{F} 4 / 80^{+}$ macrophages in the hematopoietic system of adult mice. Early studies of the physiological role of MFG-E8 emphasized cellular interactions in the innate immune system and clearance of apoptotic cells. ${ }^{8,27,40}$ Obvious phenotypic defects were not reported in developing MFG-E8 null mice, and detailed investigations were not performed on hematopoiesis. ${ }^{7,9,25,27}$ Recent studies have shown that MFG-E8 is also expressed in many other tissue types besides immune cells, but expression during hematopoiesis was not investigated. ${ }^{11-13,41,42}$ Our data clearly show that MFG-E8 is expressed at different hematopoietic sites, including the yolk sac, aorta, placenta and fetal liver during embryogenesis, but not in the adult bone marrow. Previous studies showed that developmental endothelial locus1 , which shared close structural and functional homologies, was detected in a subset of round hematopoietic cells in the yolk sac blood islands. ${ }^{43}$ This study also found that developmental endothelial locus-1 was expressed in endothelial cells, 

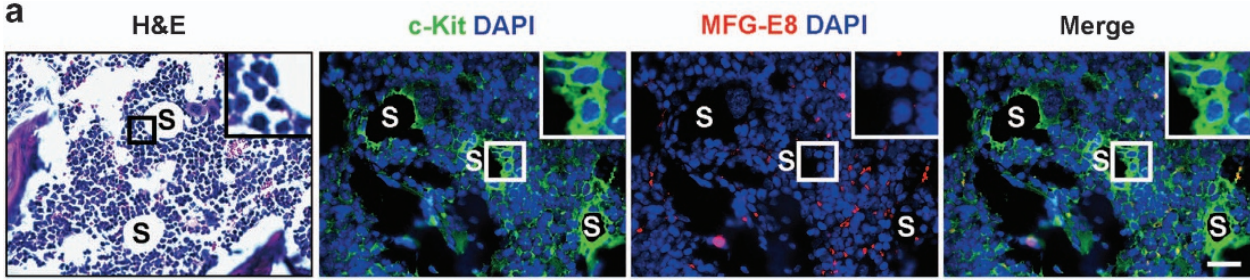

b F4/80 DAPI

MFG-E8 DAPI Merge
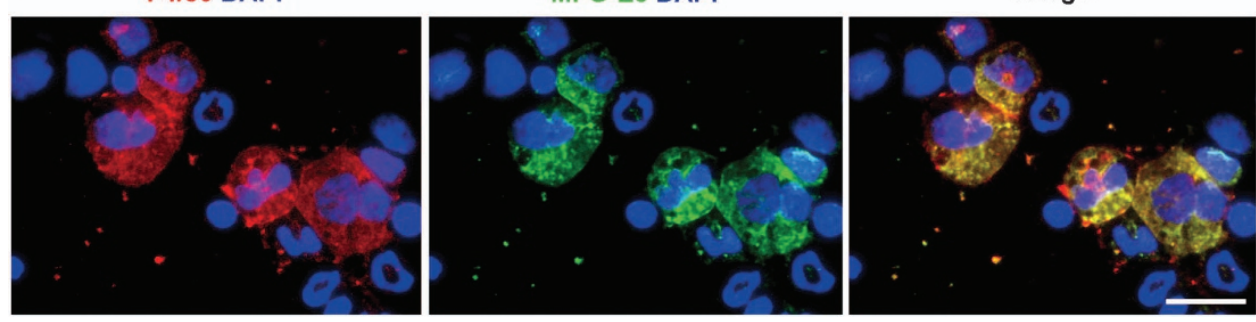

Figure 5 Milk fat globule-EGF-factor 8 (MFG-E8) expression in adult bone marrow. (a) Immunohistochemical analysis of bone marrow sections with antibodies against c-kit and MFG-E8. Note that c-kit ${ }^{+}$cells are located in the near sinusoids and these cells did not express MFG-E8. S, sinusoid. Enlarged images of the boxed region are shown in the upper right corner of each panel. (b) Expression of MFG-E8 in $\mathrm{F} 4 / 80^{+}$macrophages in the bone marrow of adult mice. Scale bar: $\mathbf{a}=50 \mu \mathrm{m} ; \mathbf{b}=20 \mu \mathrm{m}$.

but that expression declined after endothelial cells formed blood vessels, suggesting a potential role in vascular remodeling. Our data revealed that MFG-E8 was specifically expressed in $\mathrm{CD}_{3} 4^{+}$HSCs within blood islands. Importantly, del1 and MFG-E8 both contained EGF-like domains, with an RGD motif and discoidin-like domains. Thus, it can be speculated that MFG-E8 expressed in CD34 ${ }^{+}$cells may support growth of new vessels in the early stages of embryonic development. Indeed, recent studies have reported a potential role for MFG-E8 in modulating neovascularization. ${ }^{15}$

Because MFG-E8 was detected at all hematopoietic sites, i.e., the yolk sac, AGM, placenta, and liver in developing embryos, it may be involved in formation of specialized niches that support HSC self-renewal, differentiation and migration. Before primitive vascular networks are established, mesodermal cells in the primitive streak migrate into multiple hematopoietic sites, including the yolk sac, AGM and/or placenta, upon stimulation with vascular endothelial growth factor, Indian Hedgehog, and bone morphogenetic protein $4 .{ }^{44-46}$ It has been suggested that hematopoietic clusters in the aortic mesenchyme and the ventral walls of adjacent large vessels migrate through the endothelium and are released into the circulation. ${ }^{47}$ Fetal HSCs actively proliferate and migrate to provide an adequate supply of HSCs for postnatal life. ${ }^{48}$ In particular, AGM-derived HSCs migrate into the fetal liver and are colonized by rapid expansion at $\sim$ E15.5. ${ }^{24}$ Recent studies have suggested that MFG-E8 may have a role in acquisition of motile phenotypes, and in regulating proliferation in various cell types. In the adult hematopoiesis, however, HSCs reside in the bone marrow and closely interact with osteogenic, adipogenic and chondrogenic cells derived from the mesenchymal stem cells, continuously producing different types of blood cells. ${ }^{24,49}$ Thus, the different expression of MFG-E8 between fetal and adult HSCs may be owing to the different microenvironments surrounding them.
This speculation is further supported by other findings that MFG-E8 promoted the migration of tumor cells, cancer stem cells and non-transformed epithelial cells, by activating twist, snail, cyclins D1/D3 and mitogen-activated protein kinases. ${ }^{1,5,14,50}$ Therefore, future studies using an in vitro hematopoietic modeling system and gene silencing technology will be required for determining whether MFG-E8 involves in migration and/or proliferation of fetal HSCs.

Enucleation of erythroblasts is a critical step in differentiation of mammalian HSCs into mature erythrocytes. This event occurs between E14.5 and E16.5 in the mouse fetal liver during definitive erythropoiesis. ${ }^{51}$ Although the exact mechanism of enucleation has yet to be clarified, the nuclei of erythroblasts are polarized to one side of the cell, and extrude with a thin rim of cytoplasm and an enveloping plasma membrane, that is referred to as the 'pyrenocyte'. ${ }^{51}$ This pyrenocyte is then engulfed by a macrophage, because of the expression of phosphatidylserine on its surface, which acts as an 'eat me' signal for apoptotic cells. ${ }^{40}$ Because MFG-E8 harbors both the discoidin domain that binds to phosphatidylserine on prenocytes, and an RGD motif that can interact with macrophage integrins, MFG-E8 may act as a bridging molecule that promotes nuclei removal from erythroblasts. Because primitive erythroid cells leave the yolk sac and enter circulation as nucleated cells before enucleation, it is unlikely that MFG-E8 expressed in the yolk sac is involved in the enucleation process. However, we cannot exclude a presumptive role for MFG-E8 in enucleation in the fetal liver of mouse embryos.

One of the murine fetal HSC markers, CD34, is known to be expressed in particular hematopoietic progenitors, such as short-term HSCs, multipotent progenitors, common myeloid progenitors and granulocyte-macrophage progenitors. ${ }^{52,53}$ The short-term HSCs and the multipotent progenitors are transient 
hematopoietic cells that generate all lineages. Our data showed that $\mathrm{CD} 34^{+}$cells purified from the mouse liver expressed the additional hematopoietic markers, c-Kit and Sca-1. Furthermore, we also demonstrated that purified $\mathrm{CD} 34^{+}$cells, but not CD34- ${ }^{-}$cells, highly expressed MFG-E8 mRNA. These result strongly suggested that $\mathrm{CD} 34^{+}$HSCs expressed MFG-E8, although the potential role of MFG-E8 in the embryonic hematopoiesis remains to be elucidated.

MFG-E8 has attracted much attention from many different biomedical fields, owing to its multifaceted roles in diverse pathophysiological events. In this study, we showed, for the first time, that MFG-E8 was expressed in multiple hematopoietic tissues of developing mouse embryos. Future investigations into the exact physiological roles and the action mechanism of MFG-E8 during hematopoiesis are therefore important directions for future research.

\section{CONFLICT OF INTEREST}

The authors declare no conflict of interest.

\section{ACKNOWLEDGEMENTS}

This work was supported by The Ministry of Science, ICT \& Future Planning (MSIP) of the National Research Foundation of Korea (No. 2012M3A9B4028636 and No. 2012M3A9C7050139) and by Korea University Grant.

1 Carrascosa C, Obula RG, Missiaglia E, Lehr HA, Delorenzi M, Frattini M et al. MFG-E8/lactadherin regulates cyclins D1/D3 expression and enhances the tumorigenic potential of mammary epithelial cells. Oncogene 2012; 31: 1521-1532.

2 Ensslin MA, Shur BD. Identification of mouse sperm SED1, a bimotif EGF repeat and discoidin-domain protein involved in sperm-egg binding. Cell 2003; 114: 405-417.

3 Ye H, Li B, Subramanian V, Choi BH, Liang Y, Harikishore A et al. NMR solution structure of C2 domain of MFG-E8 and insights into its molecular recognition with phosphatidylserine. Biochim Biophys Acta 2013; 1828: 1083-1093.

4 Andersen MH, Berglund L, Rasmussen JT, Petersen TE. Bovine PAS-6/7 binds alpha $v$ beta 5 integrins and anionic phospholipids through two domains. Biochemistry 1997; 36: 5441-5446.

5 Ensslin MA, Shur BD. The EGF repeat and discoidin domain protein, SED1/MFG-E8, is required for mammary gland branching morphogenesis. Proc Natl Acad Sci USA 2007; 104: 2715-2720.

6 Hanayama R, Tanaka M, Miwa K, Shinohara A, Iwamatsu A, Nagata S. Identification of a factor that links apoptotic cells to phagocytes. Nature 2002; 417: 182-187.

7 Ait-Oufella H, Kinugawa K, Zoll J, Simon T, Boddaert J, Heeneman S et al. Lactadherin deficiency leads to apoptotic cell accumulation and accelerated atherosclerosis in mice. Circulation 2007; 115: 2168-2177.

8 Akakura S, Singh S, Spataro M, Akakura R, Kim JI, Albert ML et al. The opsonin MFG-E8 is a ligand for the alphavbeta5 integrin and triggers DOCK180-dependent Rac1 activation for the phagocytosis of apoptotic cells. Exp Cell Res 2004; 292: 403-416.

9 Atabai K, Fernandez R, Huang X, Ueki I, Kline A, Li Y et al. Mfge8 is critical for mammary gland remodeling during involution. Mol Biol Cell 2005; 16: 5528-5537.

10 Fuller AD, Van Eldik LJ. MFG-E8 regulates microglial phagocytosis of apoptotic neurons. J Neuroimmune Pharm 2008; 3: 246-256.

11 Nandrot EF, Anand M, Almeida D, Atabai K, Sheppard D, Finnemann SC. Essential role for MFG-E8 as ligand for alphavbeta5 integrin in diurnal retinal phagocytosis. Proc Natl Acad Sci USA 2007; 104: 12005-12010.
12 Atabai K, Jame S, Azhar N, Kuo A, Lam M, McKleroy W et al. Mfge8 diminishes the severity of tissue fibrosis in mice by binding and targeting collagen for uptake by macrophages. J Clin Invest 2009; 119: 3713-3722.

13 Bu HF, Zuo XL, Wang X, Ensslin MA, Koti V, Hsueh W et al. Milk fat globule-EGF factor $8 /$ lactadherin plays a crucial role in maintenance and repair of murine intestinal epithelium. J Clin Invest 2007; 117: 3673-3683.

14 Jinushi M, Nakazaki Y, Carrasco DR, Draganov D, Souders N, Johnson M et al. Milk fat globule EGF-8 promotes melanoma progression through coordinated Akt and twist signaling in the tumor microenvironment. Cancer Res 2008; 68: 8889-8898.

15 Neutzner M, Lopez T, Feng X, Bergmann-Leitner ES, Leitner WW, Udey MC. MFG-E8/lactadherin promotes tumor growth in an angiogenesis-dependent transgenic mouse model of multistage carcinogenesis. Cancer Res 2007; 67: 6777-6785.

16 Silvestre JS, Thery C, Hamard G, Boddaert J, Aguilar B, Delcayre A et al. Lactadherin promotes VEGF-dependent neovascularization. Nat Med 2005; 11: 499-506.

17 Weissman IL. Stem cells: units of development, units of regeneration, and units in evolution. Cell 2000; 100: 157-168.

18 Travlos GS. Normal structure, function, and histology of the bone marrow. Toxicol Pathol 2006; 34: 548-565.

19 Wilson A, Trumpp A. Bone-marrow haematopoietic-stem-cell niches. Nat Rev Immunol 2006; 6: 93-106.

20 Yoder MC, Hiatt K, Dutt P, Mukherjee P, Bodine DM, Orlic D. Characterization of definitive lymphohematopoietic stem cells in the day 9 murine yolk sac. Immunity 1997; 7: 335-344.

21 Yoder MC, Hiatt K, Mukherjee P. In vivo repopulating hematopoietic stem cells are present in the murine yolk sac at day 9.0 postcoitus. Proc Nat Acad Sci USA 1997; 94: 6776-6780.

22 Gekas C, Dieterlen-Lievre F, Orkin SH, Mikkola HK. The placenta is a niche for hematopoietic stem cells. Dev Cell 2005; 8: 365-375.

23 Ottersbach K, Dzierzak E. The murine placenta contains hematopoietic stem cells within the vascular labyrinth region. Dev Cell 2005; 8: 377-387.

24 Mikkola HK, Orkin SH. The journey of developing hematopoietic stem cells. Development 2006; 133: 3733-3744.

25 Boddaert J, Kinugawa K, Lambert JC, Boukhtouche F, Zoll J, Merval R et al. Evidence of a role for lactadherin in Alzheimer's disease. Am J Pathol 2007; 170: 921-929.

26 Hanayama R, Nagata S. Impaired involution of mammary glands in the absence of milk fat globule EGF factor 8. Proc Natl Acad Sci USA 2005; 102: 16886-16891.

27 Hanayama R, Tanaka M, Miyasaka K, Aozasa K, Koike M, Uchiyama Y et al. Autoimmune disease and impaired uptake of apoptotic cells in MFG-E8deficient mice. Science 2004; 304: 1147-1150.

28 Miksa M, Wu R, Dong W, Komura H, Amin D, Ji $\mathrm{Y}$ et al. Immature dendritic cell-derived exosomes rescue septic animals via milk fat globule epidermal growth factor-factor VIII [corrected]. J Immunol 2009; 183: 5983-5990.

29 Catherine R, Katrin O, Elaine D. Hematopoietic Stem Cells during Embryonic Development. Landes Bioscience: London, England, 2006.

30 Dzierzak E, Speck NA. Of lineage and legacy: the development of mammalian hematopoietic stem cells. Nat Immunol 2008; 9: 129-136.

31 Ema M, Yokomizo T, Wakamatsu A, Terunuma T, Yamamoto M, Takahashi S. Primitive erythropoiesis from mesodermal precursors expressing VEcadherin, PECAM-1, Tie2, endoglin, and CD34 in the mouse embryo. Blood 2006; 108: 4018-4024.

32 Guo Y, Lubbert M, Engelhardt M. CD34- hematopoietic stem cells: current concepts and controversies. Stem cells 2003; 21: 15-20.

33 McKinney-Freeman SL, Naveiras O, Yates F, Loewer S, Philitas M, Curran $\mathrm{M}$ et al. Surface antigen phenotypes of hematopoietic stem cells from embryos and murine embryonic stem cells. Blood 2009; 114 : 268-278.

34 Ogawa M, Tajima F, Ito T, Sato T, Laver JH, Deguchi T. CD34 expression by murine hematopoietic stem cells. Developmental changes and kinetic alterations. Ann NY Acad Sci 2001; 938: 139-145.

35 Taoudi S, Morrison AM, Inoue H, Gribi R, Ure J, Medvinsky A. Progressive divergence of definitive haematopoietic stem cells from the endothelial compartment does not depend on contact with the foetal liver. Development 2005; 132: 4179-4191. 
36 Young PE, Baumhueter S, Lasky LA. The sialomucin CD34 is expressed on hematopoietic cells and blood vessels during murine development. Blood 1995; 85: 96-105.

37 Palis J, Yoder MC. Yolk-sac hematopoiesis: the first blood cells of mouse and man. Exp Hematol 2001; 29: 927-936.

38 Osawa M, Hanada K, Hamada H, Nakauchi H. Long-term Iymphohematopoietic reconstitution by a single CD34-low/negative hematopoietic stem cell. Science 1996; 273: 242-245.

39 Nombela-Arrieta C, Pivarnik G, Winkel B, Canty KJ, Harley B, Mahoney JE et al. Quantitative imaging of haematopoietic stem and progenitor cell localization and hypoxic status in the bone marrow microenvironment. Nat Cell Biol 2013; 15: 533-543.

40 Yoshida H, Kawane K, Koike M, Mori Y, Uchiyama Y, Nagata S. Phosphatidylserine-dependent engulfment by macrophages of nuclei from erythroid precursor cells. Nature 2005; 437: 754-758.

41 Aoki N, Ishii T, Ohira S, Yamaguchi Y, Negi M, Adachi T et al. Stage specific expression of milk fat globule membrane glycoproteins in mouse mammary gland: comparison of MFG-E8, butyrophilin, and CD36 with a major milk protein, beta-casein. Biochim Biophys Acta 1997; 1334: 182-190.

42 Franchi A, Bocca S, Anderson S, Riggs R, Oehninger S. Expression of milk fat globule EGF-factor 8 (MFG-E8) mRNA and protein in the human endometrium and its regulation by prolactin. Mol Hum Reprod 2011; 17: 360-371.

43 Hidai C, Zupancic T, Penta K, Mikhail A, Kawana M, Quertermous EE et al. Cloning and characterization of developmental endothelial locus-1: an embryonic endothelial cell protein that binds the alphavbeta3 integrin receptor. Gene Dev 1998; 12: 21-33.

44 Baron M. Induction of embryonic hematopoietic and endothelial stem/progenitor cells by hedgehog-mediated signals. Differentiation 2001; 68: 175-185.

45 Sadlon TJ, Lewis ID, D'Andrea RJ. BMP4: its role in development of the hematopoietic system and potential as a hematopoietic growth factor. Stem Cells 2004; 22: 457-474.

46 Shalaby F, Ho J, Stanford WL, Fischer KD, Schuh AC, Schwartz L et al. A requirement for $\mathrm{Flkl}$ in primitive and definitive hematopoiesis and vasculogenesis. Cell 1997; 89: 981-990.
47 North TE, de Bruijn MF, Stacy T, Talebian L, Lind E, Robin C et al. Runx1 expression marks long-term repopulating hematopoietic stem cells in the midgestation mouse embryo. Immunity 2002; 16: 661-672.

48 Lessard J, Faubert A, Sauvageau G. Genetic programs regulating HSC specification, maintenance and expansion. Oncogene 2004; 23 7199-7209.

49 Mendes SC, Robin C, Dzierzak E. Mesenchymal progenitor cells localize within hematopoietic sites throughout ontogeny. Development 2005; 132 : 1127-1136.

50 Jinushi M, Chiba S, Yoshiyama H, Masutomi K, Kinoshita I, Dosaka-Akita H et al. Tumor-associated macrophages regulate tumorigenicity and anticancer drug responses of cancer stem/initiating cells. Proc Natl Acad Sci USA 2011; 108: 12425-12430.

51 McGrath KE, Kingsley PD, Koniski AD, Porter RL, Bushnell TP, Palis J. Enucleation of primitive erythroid cells generates a transient population of "pyrenocytes" in the mammalian fetus. Blood 2008; 111 2409-2417.

52 Chotinantakul K, Leeanansaksiri W. Hematopoietic stem cel development, niches, and signaling pathways. Bone Marrow Res 2012; 2012: 270425.

53 van Galen P, Kreso A, Mbong N, Kent DG, Fitzmaurice T, Chambers JE et al. The unfolded protein response governs integrity of the haematopoietic stem-cell pool during stress. Nature 2014; 510: 268-272.

(i) (5) (2) This work is licensed under a Creative Commons Attribution-NonCommercial-ShareAlike $\quad \mathbf{4 . 0}$

International License. The images or other third party material in this article are included in the article's Creative Commons license, unless indicated otherwise in the credit line; if the material is not included under the Creative Commons license, users will need to obtain permission from the license holder to reproduce the material. To view a copy of this license, visit http://creativecommons.org/licenses/by-nc-sa/4.0/

Supplementary Information accompanies the paper on Experimental \& Molecular Medicine website (http://www.nature.com/emm) 